\title{
PERFIL PROFESIONAL DEL INGENIERO AGRÍCOLA EN COSTA RICA
}

\author{
Herminia Casanueva López \\ Docente de la Escuela de Ingeniería Agrícola de la Universidad de Costa Rica.
}

Recibido 04-XI-2004 • Aceptado 07-XII-2004

\begin{abstract}
Resumen: La Ingeniería Agrícola, como carrera y como profesión, existe en Costa Rica desde 1974, por lo que se le considera una profesión joven, sin embargo existe un número ya significativo de profesionales que han ido ubicándose en las distintas áreas propias de su quehacer, por lo que el momento es propicio para realizar una sistematización explícita de las áreas de desempeño profesional, las actividades que se desarrollan en cada área y la proyección de nuevas áreas y actividades para los próximos años.

Para la realización de este trabajo, se aplicó una metodología diseñada para "sistemas suaves" y que se caracteriza por ser participativa y flexible por lo que responde metodológicamente a la naturaleza del proyecto.

Como producto de la investigación se identificaron siete grandes áreas del quehacer profesional y diez actividades, que se intersecan con las áreas, de esta matriz se genera el perfil profesional actual del Ingeniero Agrícola en Costra Rica y que permite que futuros proyectos lo tomen como base conforme la Ingeniería Agrícola y su práctica profesional evolucionen.
\end{abstract}

Palabras clave: Perfil profesional, Sistemas Suaves, Ingeniería Agrícola.

\section{Antecedentes}

La Ingeniería Agrícola en Costa Rica, como carrera universitaria y como profesión es relativamente joven, sin embargo se cuenta ya con número significativo de profesionales estudiantes que permiten establecer el perfil profesional de esta área.

Propuesta como carrera interdisciplinaria entre las Facultades de Ingeniería y Agronomía el 24 de setiembre de 1973 en la sesión número 1964-11, por el Consejo Universitario, a solicitud del decano de Ingeniería Ing. Walter Sagot, se autoriza el inicio de labores mediante resolución 109 de la Vicerrectoría de Docencia en diciembre de 1976 y se aprueba el primer plan de estudios para impartir la Licenciatura en Ingeniería Agrícola (Archivo Consejo Universitario. Universidad de Costa Rica).

La carrera inicia operaciones ofreciendo cursos principalmente en las áreas de Riego y Drenaje, Maquinaria Agrícola y Construcciones Rurales, al momento de su creación es una opción profesional nueva, que no significa duplicidad con respecto al ejercicio de ninguna otra profesión en el país e incluso en la región centroamericana.

Con el inicio de actividades se hace evidente la necesidad de contar con la autonomía y apoyo administrativo necesarios para el buen desarrollo de la academia por lo que se inician las gestiones para crear la Escuela de Ingeniería Agrícola 


\begin{abstract}
Agriculture engineering either as a university major or as a profession has existed in Costa Rica since 1974. Thus, it is basically considered a young field of study. However, a meaningful number of professionals exist who have been filling positions in different areas of this field. Thus, an explicit systematisation must be done of the areas of professional performance, the activities developed in each area and the influence of new areas and activities in coming years. To conduct this study, a methodology used for "soft systems" was used. It is characterized by being participative and flexible. Thus, it responds in a methodological way to the nature of the project. As a result of the research study, seven large areas of the profession and ten activities which overlap with the areas were identified. Along these lines, the present professional profile is generated for the agricultural engineer in Costa Rica. The profile can be used as a point of departure for prospective projects as agricultural engineering and its professional practice evolve.
\end{abstract}

Key words: Agricultural engineering, explicit systematisation, professional performance, soft systems. como unidad académica en la Facultad de Ingeniería (Archivo Escuela de Ingeniería Agrícola. Universidad de Costa Rica).

Después de 10 años de operación, finalmente la Asamblea Colegiada Representativa aprueba la creación de la Escuela en la sesión número 37, artículo 4 del 19 de junio de 1986 (Archivo Consejo Universitario).

Actualmente, la carrera se ofrece en el Instituto Tecnológico y en la Universidad de Costa Rica, y se ofrecen las opciones de bachillerato y licenciatura en ambas instituciones.

El ejercicio profesional de la Ingeniería Agrícola está regido por el Colegio de Ingenieros Electricistas, Mecánicos e Industriales que incluye entre sus miembros a los ingenieros agrícolas, la modificación de la Ley Orgánica del Colegio Federado de Ingenieros y Arquitectos, para incluir a los ingenieros agrícolas, se publicó en La Gaceta del 29 de febrero de 2002 (Archivo Asociación Costarricense de Ingenieros Agrícolas).

Aunque no se cuenta con estadísticas oficiales que describan el empleo de los graduados de la Escuela, si se sabe que existe una gran demanda del mercado por los egresados, al grado que algunos reciben ofertas laborales tan atractivas que de alguna forma "compiten" con la finalización de sus proyectos de graduación.

Es este, entonces un buen momento para establecer el perfil profesional del Ingeniero Agrícola en Costa Rica, como herramienta útil y oportuna para proyectar el quehacer académico de los próximos años, aportar a la asociación de profesionales un marco de referencia importante para cumplir su mandato ante la sociedad y sus agremiados y finalmente para ponerlo a disposición del sector agroindustrial del país, de los profesionales afines y de todos aquellos que se interesen por conocer este campo profesional. 


\section{Objetivo general}

Establecer el Perfil Profesional del Ingeniero Agrícola en Costa Rica.

\subsection{Objetivos específicos}

1. Identificar los ámbitos de desempeño profesional del Ingeniero Agrícola.

2. Identificar las funciones que comprende el proceso seguido por la Ingeniería Agrícola en la realización de las tareas que le son propias.

3. Identificar y cuantificar los usuarios actuales y potenciales de los servicios que ofrece el Ingeniero Agrícola.

4. Valorar la función estratégica que el Ingeniero Agrícola puede jugar en el desempeño del sector agroindustrial del país.

\section{Marco teórico metodológico}

Para realizar este perfil profesional se aplicó una versión simplificada de la Metodología para Sistemas Suaves, propuesta por P. Checkland, que ya había sido utilizada por la autora con buen éxito en trabajos anteriores (Casanueva: 1985, 1987).

Esta técnica es ampliamente utilizada para el análisis de sistemas sociales con situaciones problemáticas no estructuradas o no claramente definidas, en contraposición a los sistemas productivos o "duros" como los califican algunos autores, permite mayor flexibilidad y hace a los participantes actores responsables de la conducción del proceso y de sus resultados (Couprie: 2001).

Los llamados problemas duros son aquellos que se caracterizan por el hecho de poder ser claramente definidos. Se asume que existe una solución unívoca y que se puede definir un número específico de metas intermedias que pueden antecederla. En esencia, con un problema duro se puede definir qué tipo de logro se alcanzará antes de implementar la solución. El "QUÉ” y el "CÓMO" de un problema duro pueden ser determinados en las primeras etapas del proceso.

Los sistemas suaves, por el contrario, son difíciles de definir. Ellos pueden tener un gran número de componentes sociales. Cuando se piensa en un sistema suave, no se piensa en un problema, sino en una situación problemática, se conoce que unas situaciones o procesos no están funcionando en la manera en que se desea que lo hagan, se busca la razón de esto y las posibles alternativas para que el proceso ocurra de la manera en que se desea.

Los sistemas suaves no pueden ser asumidos o intervenidos como si fuesen parte del dominio de la realidad objetiva; el diseño o la modelación de un sistema social debe considerar la variedad de puntos de vista que coexisten en su interior. Las personas que participan en un sistema estructuran sus acciones en función del punto de vista que tienen sobre dicho sistema, por eso se puede afirmar que: "La metodología de sistemas suaves comienza cuando vemos el mundo con los ojos del otro" (Churchman, 1968, p. 23).

Cuando se pretende conocer la experiencia ajena, se ha de considerar que sólo se la puede conocer a través de los conceptos que los actores participantes del sistema manifiesten y de sus conductas.

El reconocimiento de la perspectiva ajena se constituyó de hecho en medio y fin de un movimiento dentro de la Teoría General de Sistemas, que gracias al reconocimiento acerca de las limitaciones e insatisfactoria práctica con los métodos derivados de los sistemas duros, aplicados en sistemas sociales, lanzó una propuesta de sistemas con una visión humanista (interpretativa).

Para los proponentes de esta metodología una explicación siempre es una proposición que reformula o recrea las relaciones entre los elementos componentes de un fenómeno. Al aproximarse el estudio de un sistema desde las relaciones entre sus componentes, en contraposición a los 
modelos que buscan la explicación en la identificación de los componentes, se preserva el carácter dinámico que caracteriza los sistemas y la evolución dinámica que les es inherente (Maturana, 1972, p. 14).

Así, los criterios de validación que se construyen permanentemente al interior de los sistemas sociales, ajustan y moldean la conducta de sus miembros permitiendo la evolución cultural de una sociedad o de un grupo determinado dentro de ella.

Este breve resumen de las principales características de la metodología de sistemas suaves muestra la importancia que la perspectiva sistémica tiene para la comprensión de los fenómenos sociales (educativos, políticos, económicos o culturales), al proponer una metodología humanista que incorpora la percepción de los actores, dinámica e inclusiva que ofrece una herramienta útil para la investigación social.

La primera referencia a sistemas suaves, fue hecha por Checkland en 1975, el modelo elaborado por este autor describe 7 etapas para la superación de una situación problemática. Tal como aparece en la figura, hay una secuencia lógica de las etapas que va del 1 al 7, pero es posible empezar en cualquier punto del proceso, dependiendo de la situación concreta: también es esencial para el modelo la posibilidad de volver atrás en cualquier punto, así como la interacción de las partes. De hecho los más efectivos analistas de sistemas trabajan simultáneamente en varios niveles.

La metodología contiene dos clases de actividades: los estadios 1, 2, 5, 6 у 7 pertenecen al mundo real por lo que necesariamente incluyen personas en la situación problemática analizada. Los estadios 3,4 , 4a y 4 b, son representaciones conceptuales de dicha realidad que se elaboran a partir de lo que los actores del proceso manifiestan, no se parte de ninguna definición predeterminada, permitiendo que la elaboración a la que se llegue refleje lo más fielmente posible las distintas concepciones que se tienen sobre el sistema.
La selección de la metodología se fundamenta en que por sus características se ajusta a los propósitos de la investigación, esto es a identificar los sistemas sobre los que opera la ingeniería agrícola y las actividades que dentro de estos sistemas se ejecutan, identificando también las relaciones entre las actividades al interior de cada sistema.

En el caso de la identificación de un perfil profesional de una carrera relativamente joven y que no ha sido definido anteriormente para un contexto específico, como es este caso, se debe empezar por hacer explícitas las definiciones y modelos conceptuales que los diferentes actores han formado en sus mentes, a partir de sus experiencias y aprendizajes, para a partir de estas definiciones buscar las semejanzas y las diferencias y construir con ellos definiciones de consenso.

Para la aplicación de esta técnica a la consulta de expertos se involucró a los distintos actores que aparecieron en el proceso, sus relaciones con el sistema en estudio y las elaboraciones conceptuales que cada uno de ellos tiene, tal como la metodología seleccionada propone (Checkland, 1981).

Con las definiciones elaboradas y validadas por los mismos actores se volverá a la situación real, que describe el autor y mediante una técnica de FODA se establecerá la validación final del perfil profesional.

\section{Delimitación del objeto de estudio}

Para este proyecto se definen sus alcances como: Elaborar el Perfil Profesional del Ingeniero Agrícola en Costa Rica, con un nivel de formación de bachillerato universitario generalista.

Las tendencias más actuales señalan la preferencia de los estudiantes por la opción de bachillerato frente a la licenciatura, principalmente por razones de tiempo y el hecho de que ambas les permiten, el 


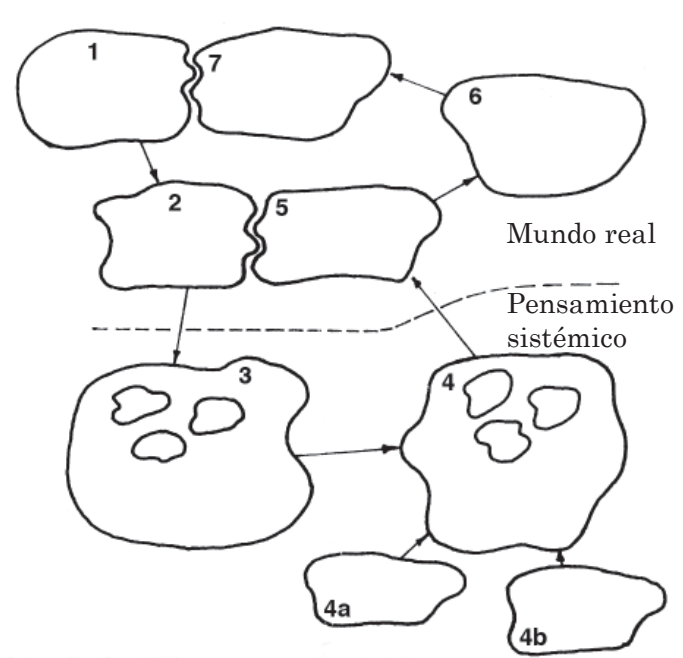

Fig. 1. Esquema Metodológico Básico. Checkland, 1975.

Simbología de la Figura:

1. Situación problemática no estructurada.

2. El problema: situación expresada.

3. Definiciones fundamentales del sistema relevante.

4. Modelo Conceptual.

4a. Sistema conceptual formal.

4b. Otros sistemas pensados.

5. Comparación de 4 con 2 .

6. Cambios posibles.

7. Acciones para superar la situación problemática.

ingreso a programas de maestría en igualdad de circunstancias.

En cuanto a la formación generalista, la Asamblea de Escuela, cuando aprobó el programa de bachillerato actualmente vigente* consideró que a nivel de bachillerato no es posible ni conveniente diferenciar las áreas ni mucho menos establecer énfasis curriculares, y que lo más conveniente es ofrecer una formación generalista con los componentes fundamentales de las áreas de aplicación.

\section{Recolección de la información}

\subsection{Conceptos básicos}

De acuerdo con la metodología propuesta se procedió a iniciar la recolección de la "percepción" que de la Ingeniería Agrícola tienen los docentes de la escuela, considerados para estos efectos como panel de expertos, para lo que se distribuyó el cuestionario $\mathrm{N}^{\circ} 1$ (anexo 1), el cual fue contestado por la totalidad de los profesores, incluyendo a los interinos e independientemente de su jornada laboral.

El propósito de este cuestionario fue recoger los conceptos básicos y las definiciones que cada experto consultado ha elaborado a partir de sus estudios y de su experiencia profesional.

De las respuestas a este cuestionario se obtuvo la primera elaboración de conceptos básicos que en criterio del panel de expertos, definen la Ingeniería Agrícola.

También se consultó sobre las tareas, entendidas como funciones, que se desarrollan en el ejercicio profesional, así como las áreas o campos de acción sobre los que se aplican estas funciones.

\section{Conceptos generados:}

Solución de problemas agrícolas.

Integrar desarrollo tecnológico con el balance ambiental del sistema.

Calidad alimentaria.

Necesidad alimentaria.

Mejorar la producción agrícola y sus productos.

Interfaz físico-biológico. 


\section{Funciones:}

Esta primera consulta permitió obtener la lista de funciones que desempeña el Ingeniero Agrícola en su práctica profesional, independientemente de la o las áreas en que desarrolle su ejercicio profesional, las funciones identificadas fueron:

Manejo.

Planificación.

Diseño. Puesta en operación.

Adaptación.

Supervisión.

Evaluación.

Asesoría.

Modernización.

Ventas.

Desarrollo.

Elaboración

de reportes.

Administración.

Docencia.

Investigación.

Áreas de acción para las

funciones identificadas:

La tercer categoría identificada fueron los principales sistemas de referencia sobre los que se aplican o desarrollan las funciones descritas:

Riego y drenaje para cultivos agrícolas tropicales.

Conservación de suelos y aguas.

Mecanización Agrícola.

Procesamiento de productos agrícolas.

Infraestructura rural.

Tratamiento de residuos.

Tratamiento de aguas.

Biosistemas.
Sistemas agroindustriales.

Una misma función puede aplicarse a más de un sistema de referencia.

\subsection{Definición de Ingeniería Agrícola}

La siguiente etapa consistió en establecer reuniones con los grupos de docentes agrupados de acuerdo con su área de especialización o práctica profesional, en la que ellos mismos se habían ubicado, se aplicó el cuestionario $\mathrm{N}^{0} 2$ (anexo 2) y se obtuvieron las definiciones fundamentales de cada área y una definición general de Ingeniería Agrícola, generada a partir de las respuestas individuales a los cuestionarios y una sesión posterior de trabajo de grupo con la participación de los expertos consultados para obtener una definición de consenso (anexo 3).

\section{Definición de Ingeniería Agrícola}

La Ingeniería Agrícola en Costa Rica consiste en la aplicación de los conceptos básicos de la ingeniería al desarrollo de los sistemas relacionados con la producción alimenticia.

Conceptualmente la Ingeniería Agrícola tiene como referente la física aplicada a los biosistemas de producción agrícola.

La base del desarrollo de esta profesión es el Enfoque de Sistemas. Desde este punto de vista el Ingeniero Agrícola (I. A.) debe optimizar recursos y maximizar rendimientos sin descuidar la calidad de los productos en el proceso para satisfacer las exigencias del mercado.

Debe establecerse que el I. A. participa en el sistema productivo afectando elementos de este, que requieren de una transformación o adaptación basada en conceptos físicos y matemáticos de la ingeniería moderna. Elementos como el suelo, el agua, el ambiente, los equipos, el producto y sus residuos son analizados por el I. A. 


\subsection{Descripción de las áreas}

Se establecieron las siguientes áreas de aplicación y desarrollo de la Ingeniería Agrícola:

1. Área de Suelos y Aguas.

2. Área de Mecanización Agrícola

3. Área de Infraestructura Rural

4. Área de Procesamiento de Productos Agrícolas

5. Área de Protección Ambiental

6. Área de Producción Agrícola Intensiva

7. Área de Administración de Sistemas de Producción Agrícola

\subsection{1. Área de suelos y aguas}

Comprende el diseño e implementación de sistemas de manejo de riego, drenaje y conservación de suelos que siendo ecológicamente sostenibles garanticen que a cada cultivo se le brinden las condiciones hídricas y edáficas óptimas para su desarrollo, incluye las siguientes actividades:

- Determinación de las necesidades de riego y/o drenaje para su uso agrícola.

- Diseño hidráulico de canales y estructura para su uso en riego y/o drenaje.

- Diseño de sistemas de conducción de agua a presión para su uso en riego, drenaje y abastecimiento de agua en fincas e industrias agrícolas.

- Diseño de obras y selección de equipos para distribución y evacuación de agua en fincas e industrias agrícolas.
- $\quad$ Planificación, administración, operación y mantenimiento de sistemas de riego y/o drenaje.

- Avalúos y peritajes relacionados con equipos y sistemas de riego y drenaje.

- Asesoría y venta de materiales y equipos relacionados con sistemas de riego y drenaje.

- Sistemas de conservación de suelos (finca).

- Manejo de cuencas:

a. Control de erosión.

b. Control de inundaciones.

\subsection{2. Área de mecanización agrícola}

Comprende el Diseño e implementación de Sistemas de Mecanización de la Producción a nivel Agrícola y Agroindustrial sus actividades son:

Diseño, implementación y control de sistemas de mecanización.

- $\quad$ Selección y adaptación de equipos.

Acople, instalación y montaje de equipos.

- $\quad$ Mantenimiento preventivo y correctivo.

- $\quad$ Planificación y administración de sistemas mecanizados.

Asesoría y ventas de equipos y sistemas.

Avalúos y peritajes.

\subsection{3. Área de infraestructura rural}

Comprende el diseño y distribución de las obras de infraestructura a nivel de 
finca mediante la determinación de necesidades, diseño de la obra física y de los sistemas de actividades a ellos asociados. Incluye las siguientes actividades:

- $\quad$ Determinación de necesidades.

- Distribución espacial de las obras de infraestructura y soporte.

Diseño de sistemas operativos de acuerdo a las necesidades de cada finca.

- Diseño de obras de albergue (bodegas, instalaciones pecuarias o agroindustriales, habitacionales, etc.) para uso a nivel de finca.

- Diseño de estructuras de almacenamiento especiales (silos, tanques para agua, cámaras frías, etc.) para uso agrícola o agroindustrial.

Diseño de obras de cruce (puentes, alcantarillado, sifones, caminos y otras) a nivel de finca.

Diseño de obras de conservación de suelos y control de manejo de aguas de escorrentía.

- Diseño de estructuras para cultivo en ambiente controlado (viveros, germinadores, estanques para explotaciones piscícolas).

Avalúos y peritajes relacionados con establecimientos agrícolas y/o agroindustriales.

- Asesoría y venta de materiales y equipos relacionados con esta área.

- $\quad$ Mantenimiento preventivo y correctivo. Rediseño de obras de infraestructura. Rediseño de actividades.

\subsection{4. Área de procesamiento de productos agrícolas}

Comprende el diseño e implementación de sistemas agroindustriales cuyo objetivo es incrementar el valor agregado a los productos agroindustriales, comprende las siguientes actividades:

- Diseño, supervisión, manejo y operación de actividades de transporte, recibo, limpieza, clasificación, secado, conservación, almacenamiento de productos agrícolas y sus derivados.

- Diseño, selección, operación y mantenimiento de equipos y plantas de procesamiento, almacenamiento $\mathrm{y}$ conservación de productos agrícolas y sus derivados.

- Control de calidad de los procesos y certificación.

- Aseguramiento de calidad.

- Venta de materiales y equipos para sistemas de procesamiento poscosecha.

\subsection{5. Área de protección ambiental}

Comprende las actividades relacionadas con los sistemas de protección del ambiente, tanto con el mantenimiento de la sostenibilidad del entorno en los procesos de producción agrícola, así como, en el tratamiento de residuos en los procesos agroindustriales incluye las siguientes actividades:

- Diseño, implementación y control de sistemas que garanticen la sostenibilidad de los procesos de producción agrícola.

Diseño, implementación y control de sistemas de tratamiento de residuos agrícolas y agroindustriales.

- Diseño, implementación y control de sistemas de tratamiento de aguas 
residuales, tanto agrícolas como agroindustriales.

- Consultoría y participación en estudios de impacto ambiental.

- Asesoría en sistemas de protección ambiental.

\subsection{6. Área de producción agrícola intensiva}

Comprende las actividades relacionadas con los sistemas agrícolas de producción intensivos, incluye las siguientes actividades:

- Diseño, implementación y mantenimiento de viveros.

- Diseño, implementación y mantenimiento de sistemas hidropónicos de cultivo.

- Diseño, implementación de sistemas de microrriego.

- Diseño, implementación y mantenimiento de sistemas automatizados de riego y control de ambientes.

\subsection{7. Área de administración de sistemas de producción agrícola}

Comprende las actividades relacionadas con la gerencia técnica de cualquiera de los sistemas de producción, proyectos ó empresas, sean estos agrícolas o agroindustriales, es transversal a todos los ejes y funciones, incluye las siguientes actividades:

Elaboración de estudios de prefactibilidad y factibilidad de proyectos relacionados con todas las áreas de trabajo.

Administración de sistemas de producción agrícolas y agroindustriales.
Gerencia técnica de proyectos agrícolas y agroindustriales.

Evaluación, consultoría y gerenciamiento agrícola y agroindustrial.

Diseño, implementación y control de sistemas de aseguramiento de calidad.

\subsection{Frecuencia de desempeño de las funciones}

La siguiente etapa de trabajo consistió en realizar una nueva consulta de experto para identificar, a partir del ejercicio profesional que cada experto realiza, la frecuencia con que se desarrolla cada una de las funciones. En términos de la metodología utilizada esta etapa es la vuelta a lo concreto.

En esta segunda consulta de expertos, se validaron las funciones que habían sido recogidas en el primer cuestionario y se organizaron en función de su secuencia dentro del proceso productivo, generándose la siguiente lista:

1. Estudios de factibilidad

2. Diseño de obra física ó sistemas operativos

3. Selección e instalación de equipo

4. Planificación, control y administración

5. Operación y mantenimiento

6. Avalúo, peritaje y certificación

7. Asesorías y consultorías

8) Ventas

9) Docencia

10) Investigación 


\subsection{Matriz del perfil profesional}

Con la información generada hasta este momento fue posible establecer la matriz de correlación entre los sistemas conceptuales de aplicación y desarrollo de la Ingeniería Agrícola y las actividades que en la práctica profesional se ejecutan.

Como puede observarse el eje fundamental que vincula las funciones es el diseño, como tarea propia de la ingeniería, al representar conceptualmente una situación problemática, organizarla y volver a la situación concreta para transformarla, el proceso seguido por el diseño en ingeniería es el mismo que propone Checkland, en la metodología que se utilizó para elaborar el perfil pues permite reflejar de la manera más fiel la conceptualización y práctica del campo profesional que se está estudiando.

Este proceso también permitió identificar los sistemas sobre los que se aplican las funciones, considerando que ya se había definido a la ingeniería agrícola como la aplicación de los principios de la ingeniería a los sistemas productivos, era necesario identificar los sistemas propios de esta profesión y definirlos claramente.

Al cruzar las funciones con los sistemas sobre los que se aplica se generó una matriz que representa la imagen conceptual del perfil profesional estudiado. El cuadro siguiente muestra la matriz obtenida:

\section{Desempeño profesional}

Con esta matriz se procedió a elaborar el cuestionario \# 3 (anexo 4) y distribuirlo entre la mayoría de los miembros de la Asociación de Ingenieros Agrícolas, activos en ese momento y que laboraran en los distintos ámbitos del quehacer profesional, para recoger datos sobre la práctica concreta, sus datos básicos.

Una vez identificada la secuencia de las funciones y establecidos los sistemas sobre los que éstas se aplican se procedió a identificar la frecuencia de distribución de ejecución de las funciones dentro de la matriz.

El propósito de este cuestionario fue, recoger una muestra lo más amplia posible de las áreas de desempeño profesional, perfil del puesto, así como de los sistemas y funciones que desarrollan en su ejercicio profesional. Se obtuvieron 32 cuestionarios válidos y los resultados obtenidos se presentan a continuación:

\subsection{Desempeño laboral}

1.1 Labora en forma independiente 1,6\%

1.2 Labora para una empresa privada

1.3 Labora para una institución del Estado $78,5 \%$

\subsection{Distribución por área de ejercicio profesional}

1.1 Consultoría y servicios

1.2 Venta de equipos $15,6 \%$

1.3 Diseño

1.4 Construcción y ejecución $15,6 \%$

1.5 Administración

1.6 Docencia $21,8 \%$ $31,2 \%$ $18,7 \%$

1.7 Investigación $28,1 \%$ $9,3 \%$

\subsection{Grado académico}

$\begin{array}{lll}1.1 & \text { Bachillerato } & 25,0 \% \\ 1.2 & \text { Licenciatura } & 68,7 \% \\ 1.3 & \text { Maestría } & 0,16 \% \\ 1.4 & \text { Doctorado } & \end{array}$

\subsection{Frecuencia de desempeño para cada función}

En lo relativo al número de respuestas asignadas a cada función, considerada mediante la frecuencia que le fue asignada por los entrevistados, destaca que las cuatro primeras presentan una frecuencia mucho más alta que las otras, siendo de hecho las que involucran un mayor componente del tipo de diseño característico de las ingenierías.

En cuanto a las labores de docencia que también involucran diseño, este reviste 


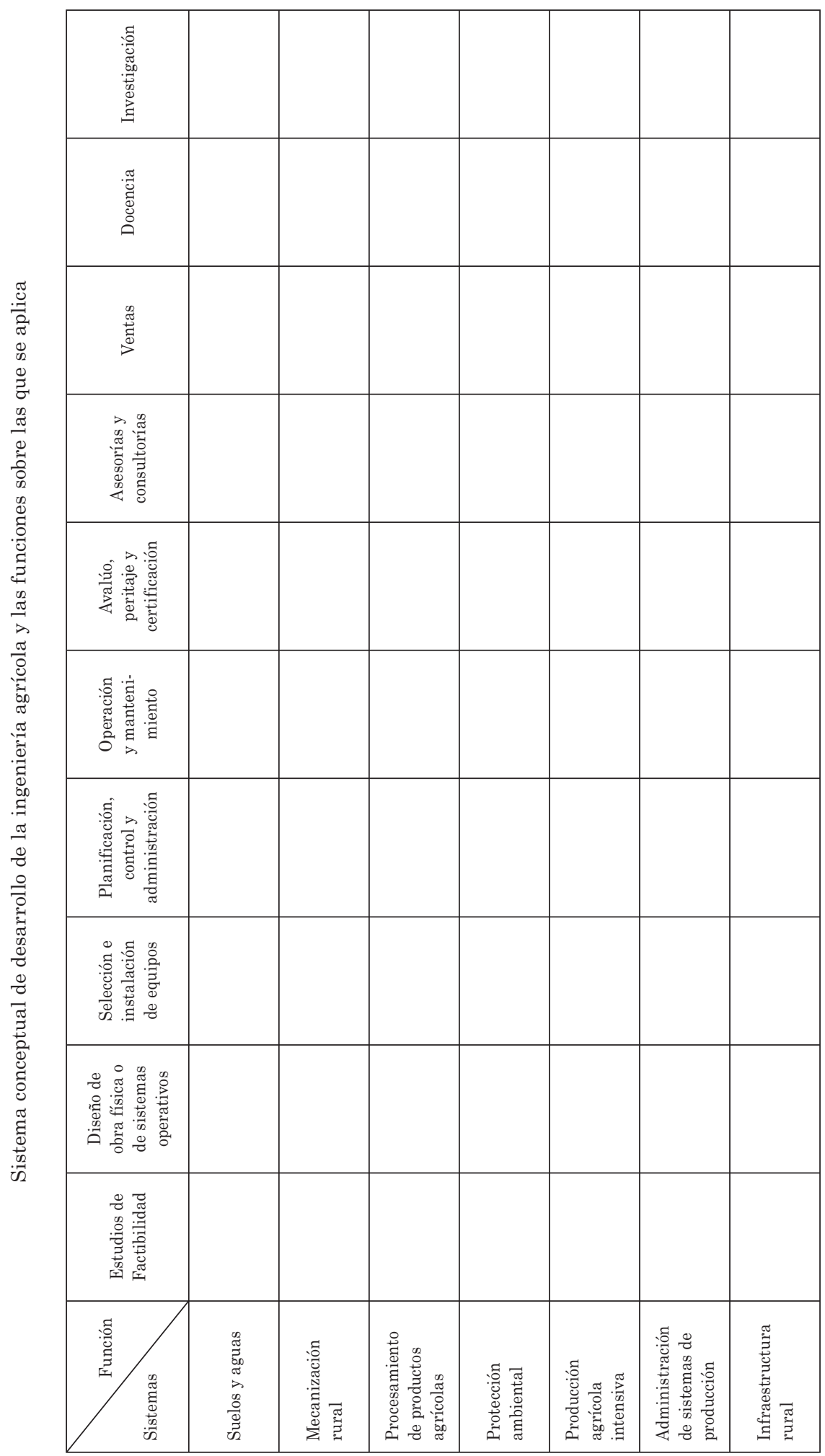


características especiales por estar orientado hacia fines didácticos, la investigación involucra diseño siempre y este puede estar orientado o no a la solución de problemas de la práctica.

El orden en que aparecen responde a la secuencia que se sigue en el proceso productivo, excepto las últimas 5 actividades que pueden incluir todo el proceso, afectándolo transversalmente o ejecutarse sobre alguna de las funciones.

Frecuencia de desempeño para cada función

\begin{tabular}{lc}
\hline Función & $\begin{array}{c}\text { Frecuencia } \\
\%\end{array}$ \\
\hline 1. Estudios de factibilidad & 14,70 \\
2. Diseño de obra física o sistemas & 21,32 \\
operativos & 13,23 \\
3. Selección e instalación de equipo & \\
4. Planificación, control y & 14,70 \\
5. Operación y mantenimiento & 10,29 \\
6. Avalúo, Peritaje y Certificación & 5,88 \\
7. Ventas & 2,20 \\
8. Docencia & 5,40 \\
9. Investigación & 5,14 \\
10. Asesoría y Consultoría & 7,35 \\
\hline
\end{tabular}

\subsection{Sistema conceptual de la Ingeniería Agrícola}

La información suministrada por los expertos al cuestionario 3, permitió llenar la matriz que se había elaborado anteriormente y que aparece en el apartado 5,5.

Al igual que para el cuadro de desempeño profesional, las cuatro primeras funciones de la matriz, presentan una frecuencia de aparición más alta y son las que involucran el mayor componente de diseño, la realización de estas funciones implica una comprensión de todo el proceso y de sus diferentes etapas para ejecutarlo correctamente. Estas etapas suponen la capacidad de visualizar el proceso como una globalidad.

El hecho de que la mayor parte de los ingenieros agrícolas que respondieron a los cuestionarios se desempeñe en áreas que implican un fuerte componente de diseño muestra que tanto la formación académica como el ejercicio profesional son ejecutados desde una visión sistémica propia de las ingenierías y que la metodología elegida para identificar el perfil profesional se ajustó a la visión y dinámica propias de esta profesión.

$\mathrm{El}$ resto de las funciones se desarrollan con menor frecuencia, pero esto no significa que sean menos importantes o que carezcan

Frecuencia de aparición de las funciones

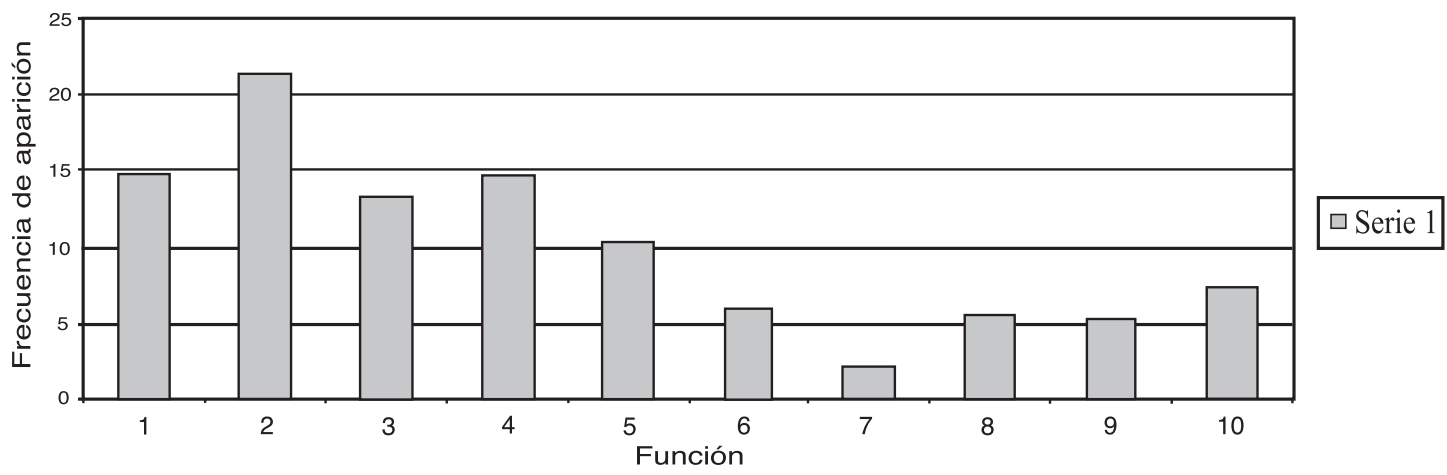




\begin{tabular}{|c|c|c|c|c|c|c|c|c|c|}
\hline 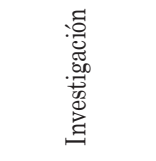 & - & $\rightarrow$ & $\neg$ & $\neg$ & $\neg$ & $\neg$ & $\neg$ & $\sim$ & $\underset{10}{H}$ \\
\hline 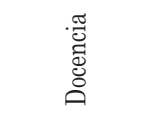 & $-r$ & $\rightarrow$ & $\neg$ & - & $r$ & $\rightarrow$ & $\rightarrow$ & $\sim$ & $\underset{10}{\overrightarrow{10}}$ \\
\hline 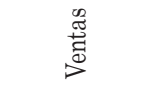 & $\rightarrow$ & 0 & $\rightarrow$ & 0 & 0 & 0 & $r$ & $\infty$ & iv \\
\hline 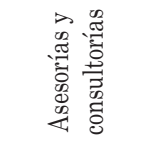 & 20 & $\rightarrow$ & 0 & $\sim$ & 0 & $\neg$ & $\neg$ & 으 & 点 \\
\hline 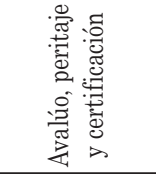 & 20 & -1 & - & 0 & 0 & 0 & $\rightarrow$ & $\infty$ & $\begin{array}{l}\infty \\
\infty \\
\infty \\
i 0^{\circ}\end{array}$ \\
\hline 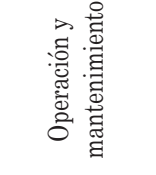 & -1 & $\infty$ & 0 & $\infty$ & 20 & $-r$ & $\neg$ & $\stackrel{\rightleftarrows}{二}$ & $\begin{array}{l}\text { बे } \\
\text { ô }\end{array}$ \\
\hline 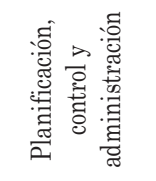 & 10 & $\rightarrow$ & $\infty$ & $\infty$ & 10 & $\infty$ & 0 & คి & $\begin{array}{l}\text { R } \\
\text { fi }\end{array}$ \\
\hline 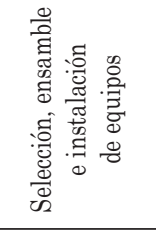 & 10 & $\sim$ & N & $\infty$ & 10 & - & 0 & $\stackrel{\infty}{-1}$ & 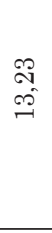 \\
\hline 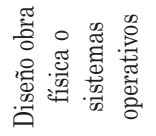 & $\sim$ & $\rightarrow$ & $\infty$ & $\infty$ & 10 & $r$ & $\sigma$ & ঞ্ণে & $\begin{array}{l}\text { مै } \\
\text { त्र }\end{array}$ \\
\hline 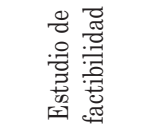 & 20 & $\neg$ & $\infty$ & $\infty$ & 10 & N & 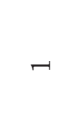 & คి & 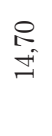 \\
\hline 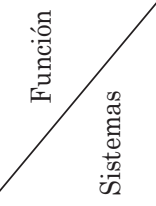 & 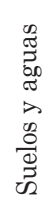 & 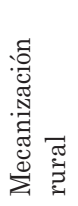 & 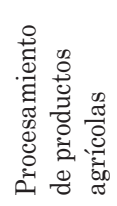 & 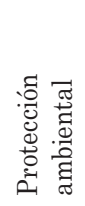 & 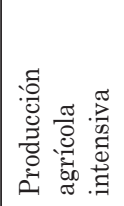 & 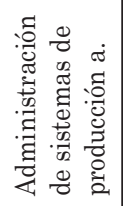 & 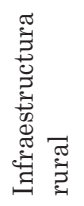 & 念 & 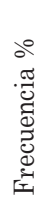 \\
\hline
\end{tabular}


de componentes de diseño, sino que este no necesariamente está asociado a una visión sistémica del proceso y que pueden desarrollarse puntualmente en forma eficiente.

Como ya se señaló las excepciones en cuanto al componente de diseño son la docencia, por su componente didáctico y la investigación que desarrollan las universidades, que puede ir dirigida a la solución de problemas prácticos e inmediatos o simplemente a ampliar el conocimiento en un campo específico.

\section{Validación final}

La última etapa del proceso de construcción del perfil profesional del ingeniero agrícola se cumplió mediante la realización de un taller convocado por la Asociación Costarricense de Ingenieros Agrícolas (ACIA) y la Escuela de Ingeniería Agrícola de la Universidad de Costa Rica, a ese taller se invitó a participar a todos los miembros de la Asociación. A los profesores y estudiantes de la carrera de Ingeniería Agrícola tanto en la Universidad de Costa Rica como en el Instituto Tecnológico de Costa Rica.

El Taller se celebró el 6 de junio del 2000 en las instalaciones del Colegio Federado de Ingenieros y Arquitectos y contó con una asistencia de más de ochenta participantes, el anexo 4 presenta la convocatoria a dicha actividad y los documentos de trabajo que la acompañaron.

El taller se convocó con tres objetivos básicos:

1. Validar el perfil profesional.

2. Aplicar un FODA para conocer la situación del ejercicio profesional actual y para los próximos años.

3. Establecer conclusiones y recomendaciones a la ACIA, la Escuela de Ingeniería Agrícola de la UCR y del ITCR, como instituciones encargadas de la formación de nuevos profesionales.

De acuerdo con el programa del taller se presentaron dos conferencias magistrales con el propósito de presentar algunas opciones y enriquecer la discusión de los temas, posteriormente se pidió a los participantes que se incorporarán a los grupos de trabajo para realizar el FODA, se organizó un grupo para cada área de ejercicio profesional. Finalmente se presentaron los resultados de los grupos a la plenaria y se aprobó el documento final.

Como consecuencia del taller se obtuvo el siguiente FODA: 
Resumen de las respuestas del análisis FODA del ejercicio profesional de la Ingeniería Agrícola en Costa Rica

\begin{tabular}{|c|c|}
\hline Fortalezas: & Oportunidades: \\
\hline $\begin{array}{l}\text { - Buena formación académica en cien- } \\
\text { cias básicas. }\end{array}$ & $\begin{array}{l}\text { - Disciplina única, es ingeniería en } \\
\text { el agro potencial de crecimiento } \\
\text { profesional. }\end{array}$ \\
\hline $\begin{array}{l}\text { - Buena formación tecnológica } \\
\text { general. }\end{array}$ & - Conciencia ambientalista en la sociedad. \\
\hline - Buen desempeño profesional. & - Carrera emergente. \\
\hline - Visión de sistema. & - Abundancia de recurso hídrico. \\
\hline - Visión integral agro industrial. & - Biodiversidad de Costa Rica. \\
\hline $\begin{array}{l}\text { - Capacidad de solucionar problemas } \\
\text { complejos. }\end{array}$ & $\begin{array}{l}\text { - Necesidad de certificadores de calidad y } \\
\text { sostenibilidad }\end{array}$ \\
\hline $\begin{array}{l}\text { - Formación que permite agregar } \\
\text { valor a los productos agrícolas. }\end{array}$ & - Pocos profesionales. \\
\hline Debilidades: & Amenazas: \\
\hline - Poca interacción entre profesionales. & - No hay políticas claras hacia el agro. \\
\hline - Falta mentalidad empresarial. & - Baja inversión económica. \\
\hline $\begin{array}{l}\text { - Faltan programas de educación } \\
\text { continua. }\end{array}$ & $\begin{array}{l}\text { - Bajos precios internacionales de } \\
\text { productos agrícolas. }\end{array}$ \\
\hline $\begin{array}{l}\text { - Falta vinculación con agro } \\
\text { industria. }\end{array}$ & - Falta infraestructura civil. \\
\hline - Enfoque inadecuado de cursos. & $\begin{array}{l}\text { - Legislación obsoleta que mantiene } \\
\text { privilegios. }\end{array}$ \\
\hline - Faltan recursos para docencia. & - Sector tradicionalista. \\
\hline $\begin{array}{l}\text { - Faltan recursos para laboratorio y } \\
\text { equipos. }\end{array}$ & - Globalización. \\
\hline - No hay énfasis específicos. & - Elevados costos de producción. \\
\hline $\begin{array}{l}\text { - Faltan opciones como maestrías o } \\
\text { especialización. }\end{array}$ & $\begin{array}{l}\text { - Invasión de otros profesionales. } \\
\text { - Desconocimiento de la profesión. }\end{array}$ \\
\hline
\end{tabular}


Como puede observarse se destacan como aspectos positivos de la profesión su visión sistémica y la buena formación científico tecnológica que ofrecen las instituciones de educación superior que imparten la carrera, existe satisfacción entre el medio por el desempeño profesional de la mayor parte de sus miembros, también se señala como aspecto positivo que es una carrera emergente y que puede dar respuestas a la problemática de la sostenibilidad.

Entre los aspectos negativos se señala la falta de recursos para la docencia, la falta de interacción entre los profesionales y una débil vinculación con el sector productivo, específicamente con la agroindustria a lo largo de la formación académica y la falta de opciones de maestría y especialización. También se señala como aspecto negativo que el sector agrícola sea muy tradicionalista y la recesión que se enfrenta y que ha obligado a postergar inversiones y mejoras.

Al finalizar el taller la plenaria aprobó las siguientes recomendaciones:

- Desarrollar un programa de actualización permanente, que abarque todas las áreas del ejercicio profesional, con actividades como talleres, seminarios y conferencias.

- Fortalecer los vínculos entre los profesionales propiciando canales de intercambio y comunicación permanentes.

- Divulgar y promover la ingeniería agrícola entre los potenciales empleadores y la sociedad en general. Elaborar material informativo como audiovisuales, folletos y afiches.

- Desarrollar un programa para promover la carrera entre los estudiantes de los últimos años de colegio. Especialmente los alumnos de los colegios técnicos agropecuarios.
- Fortalecer los vínculos y el desarrollo de programas conjuntos entre los personeros del ITCR y la UCR por ser las dos instituciones que imparten la carrera.

- Investigar nuevos campos de desarrollo para la ingeniería agrícola.

- $\quad$ Apoyar los procesos de certificación y acreditación de la carrera.

\section{Conclusiones}

La aplicación de la metodología para Sistemas Suaves de Checkland para la conceptualización del perfil profesional del ingeniero agrícola mostró que aunque sea un modelo poco conocido y aplicado en el contexto educativo, puede ser una herramienta muy útil en aquellas investigaciones en que se busque incorporar lo que Churchman llama "la visión ajena", esto es la diversidad de las percepciones de los actores individuales que conforman la visión del sistema en estudio.

En el caso de un perfil profesional que no había sido definido anteriormente en forma explícita resultó una herramienta útil pues permitió incorporar las percepciones de los actores y elaborar definiciones de consenso que fueron validadas por el colectivo, sin tener que partir de definiciones preelaboradas que podían haber contaminado o sesgado los resultados del estudio.

La elaboración de un perfil profesional debe caracterizarse por ser inclusiva, incorporando la riqueza conceptual que los actores de ese sistema en estudio han elaborado ha partir de sus experiencias, conocimientos y principalmente de las relaciones que se establecen entre ellos y el resto de la sociedad, en este sentido la metodología para sistemas suaves ofrece una opción importante de conocer y aplicar en nuevos campos.

En cuanto a los resultados del estudio se logró generar, a partir de una primera 
definición conceptual, una matriz que correlaciona los sistemas conceptuales sobre los que se aplica la Ingeniería Agrícola con las actividades o funciones que componen la práctica profesional, delimitando el ámbito de acción del profesional. Una matriz de este tipo permite que los sistemas o funciones que aparezcan como producto del desarrollo del ejercicio profesional sean fácilmente incorporadas, así como también permite que se eliminen las que eventualmente puedan desaparecer o aquellas cuyo peso disminuya a lo largo del tiempo.

Para el gremio de profesionales y para las entidades formadoras el poseer un perfil del ejercicio profesional es un elemento básico en la orientación de sus acciones. Ofrece un marco de referencia para los programas de capacitación permanente que caracterizan al profesional actual, los potenciales empleadores y profesionales afines, también para la academia es un parámetro a considerar al establecer el currículo que se desarrolla, sin embargo, el perfil profesional debe ser permanentemente revisado y ajustado a los cambios en el ejercicio profesional para que mantenga su utilidad y su vigencia, es una tarea de creación y recreación permanentes.

Este perfil profesional validado mediante un FODA planteó importantes recomendaciones para que el ingeniero agrícola pueda desempeñar eficientemente su tarea en provecho del sector agroindustrial y de la sociedad en general.

Finalmente cabe señalar que junto a la creciente complejidad del conocimiento deben establecerse vínculos que permitan una cada vez mayor interacción entre profesionales de distintos campos que contribuyan con sus saberes específicos al enriquecimiento del conocimiento, la búsqueda de metodología que aunque nacidas en otros campos del saber, pueden tener nuevas aplicaciones, es uno de los ejemplos de una práctica interdisciplinaria que debiera fomentarse.

\section{Nota}

1 Sesión celebrada el 3 de diciembre de 1997 , Acta \# 7-97 (fuente: archivo Escuela de Ingeniería Agrícola).

\section{Referencias bibliográficas:}

Archivos Asociación Costarricense de Ingenieros Agrícolas. Colegio de Ingenieros Electricistas, Mecánicos e Industriales. Colegio Federado de Inge-nieros y Arquitectos, San José. 2000.

Archivo Consejo Universitario. Universidad de Costa Rica. San José. 2000.

Archivos Escuela de Ingeniería Agrícola. Instituto Tecnológico de Costa Rica. Cartago. 2000.

Archivos Escuela de Ingeniería Agrícola. Facultad de Ingeniería. Universidad de Costa Rica. San José. 2000.

Checkland P: "Techiques in soft systems practice". Part one and two, Journal of applied systems analisis, $\mathrm{N}^{\circ} 6$, New York. 1979.

Checkland P: Systems thinking, system practice, John Willey and sons, New York. 1981.

Churchman W: "The System Approach", Dell, New York. 1968.

Couprie D: "Soft Systems Methodology". Department of Computer Science. Calgary University, 2001.

Maturana H., Varela F. "El Árbol del Conocimiento: las bases biológicas del entendimiento humano", Editorial Universitaria, Santiago, 1972. 


\section{ANEXO 1 \\ Escuela de Ingeniería Agrícola \\ PERFIL PROFESIONAL}

Setiembre, 1999

\section{Dirigida a docentes de la escuela}

Nombre Fecha

Tipo de nombramiento Jornada

Grado Académico Institución

País

Área de especialidad

Describa brevemente, para usted qué es la Ingenieria Agrícola

¿Qué hace un Ingeniero Agrícola?

¿En su práctica profesional cuáles tareas cumple? Descríbalas 


\section{ANEXO 2 \\ Escuela de Ingeniería Agrícola \\ PERFIL PROFESIONAL \\ Trabajo de grupos, por áreas}

Cuestionario \# 2

Área Fecha

Participantes:

1.- ¿Qué es Ingeniería Agrícola? (descripción de conceptos).

2.- ¿Qué hace un Ingeniero Agrícola en el área en que usted se desempeña profesionalmente? (descripción de tareas). 


\section{ANEXO 3 \\ Escuela de Ingeniería Agrícola \\ PERFIL PROFESIONAL}

Cuestionario \# 3

Dentro de la siguiente lista de áreas profesionales de la Ingeniería Agrícola, marque aquellas en las que desarrolla su práctica profesional.

Marque con un (1) la más afín, (2) para la siguiente y (3) para aquella en que se desempeña ocasionalmente:

1.- $\quad$ Manejo de Suelos y Aguas

2.- Mecanización Agrícola

3.- Poscosecha

4.- Administración

5.- Otros (especifique)

De acuerdo con el área de especialización profesional que marcó con un (1), llene el siguiente cuadro, que enlista las funciones del ingeniero agrícola.

Para contestar use las mismas claves que en el apartado anterior. (1) para la más afín, (2) para la siguiente y (3) para aquella en que se desempeña ocasionalmente, use (0) para las que no ejecuta.

\section{Funciones}

1.- $\quad$ Estudios de factibilidad

2.- Diseño

3.- Supervisión

4.- Instalación

5.- Operación y mantenimiento

6.- Administración

7.- $\quad$ Docencia

8.- Investigación

9.- Otros (especifique)

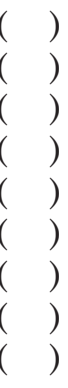




\author{
ANEXO 4 \\ Escuela de Ingeniería Agrícola \\ PERFIL PROFESIONAL \\ Encuesta a consultores externos
}

\title{
1. Ficha de identificación
}

1.1 Nombre

1.2 Edad

1.3 Grado académico

\section{Desempeño Laboral}

2.1 Trabaja en forma independiente: Posee su propia empresa

sí — no

(si su respuesta es negativa pase a 2.3)

2.2 Indique el área de especialización de su empresa:

( ) Venta de servicios

( ) Consultarías

( ) Representante de ventas

( ) Construcción

( ) Diseño

( ) Otro (describa):

Número de empleados de la empresa

Numero de profesionales

Año de fundación de la empresa

2.3 Labora para una empresa privada

sí no

(si su respuesta es negativa pase a 2.5) 
2.4 Indique el área de especialización de la empresa para la que labora

( ) Venta de servicios

( ) Consultarías

( ) Representante de ventas

( ) Construcción

( ) Diseño

( ) Otros (describa):

Puesto que ocupa (describa)

Antigüedad en el puesto que ocupa (años)

Antigüedad en la empresa (años)

2.5 Labora para una institución del Estado

sí no

Nombre de la Institución

Puesto

Antigüedad en el puesto (en años)

Antigüedad en la Institución (en años)

\section{Formación Académica}

3.1 Indique el grado mas alto que haya concluido
( ) Bachillerato
( ) Licenciatura
( ) Maestría
( ) Doctorado
( ) Especialización

3.2 Si posee un grado superior a la licenciatura indique.

( ) Título

( ) Institución

( ) Área de especialización 
4. Lea cuidadosamente entre las siguientes conceptualizaciones de la Ingeniería Agrícola y con base en su propia formación y experiencia redacte su propia definición.

- Solución de problemas agrícolas

- Integrar desarrollo tecnológico en el balance ambiental

- Manejo del ambiente físico

- Calidad alimentaria

- Necesidad alimentaria

- Mejorar la producción agrícola y los productos

- Interfaz físico-biológico

4.1 La Ingeniería Agrícola es:

4.2 A continuación se presenta una lista de actividades que un Ingeniero Agrícola realiza en su practica profesional. La segunda lista incluye los sistemas de referencia sobre los que es aplican las actividades descritas.

Lea cuidadosamente ambas listas y marque con una $\mathrm{X}$ aquellas que considera que deben formar parte del perfil del ingeniero agrícola. Marque todas las que considera convenientes y de acuerdo con su opinión agregue las que considere pertinentes.

Lista 1: Actividad desarrollada

$\begin{array}{ll}\text { Maneja } & (\text { ) } \\ \text { Diseña } & (\text { ) } \\ \text { Adapta } & (\text { ) } \\ \text { Supervisa } & (\text { ) } \\ \text { Evalúa } & (\text { ) } \\ \text { Asesora } & (\text { ) } \\ \text { Moderniza } & (\text { ) } \\ \text { Vende } & (\text { ) } \\ \text { Desarrolla } & (\text { ) } \\ \text { Elabora reportes técnicos } & (\text { ) } \\ \text { Administra } & (\text { ) } \\ \text { Enseña } & (\text { ) } \\ \text { Investiga } & (\text { ) } \\ \text { Planifica } & (\text { ) }\end{array}$


Lista 2: Sistemas de referencia

( ) Sistemas de riego y drenaje para cultivos agrícola tropicales

( ) Sistemas de conservación de suelos y aguas

( ) Sistemas de mecanización agrícola

( ) Sistemas de procesamiento de productos agrícolas

( ) Infraestructura rural

( ) Sistemas de tratamiento de residuos

( ) Sistemas de tratamiento de aguas

( ) Biosistemas

( ) Sistemas agroindustriales

( ) Sistemas intensivos en atmósferas controladas

4.3 Describa las tareas que usted ejecuta en su practica profesional. 